Revista de Psicología de la PUCP. Vol. XVII, 1, 1999.

\title{
LA PSICOFISIOLOGÍA Y EL DESARROLLO DE LA PSICOLOGÍA CLÍNICA
}

\author{
Edmundo Beteta Pacheco ${ }^{1}$ \\ Universidad Nacional Mayor de San Marcos
}

Se presentan los avances en Psicofisiología, destacando los estudios anatómicos y bioquímicos de los mecanismos cerebrales que intervienen en la conducta. Estas investigaciones permiten ampliar el campo de la Psicología Clínica, tanto en la prevención como en el diagnóstico y terapéutica de los desórdenes de la conducta. En este objetivo, el psicólogo clínico podrá realizar estudios de investigación en la comunidad con la metodología de correlacionar factores de riesgo epidemiológicos, tests neuropsicológicos y estudios por imágenes.

Palabras claves: psicofisiología, psicologia fisiológica, bases biológicas de la conducta.

The psychophisiology and the development of clinic psychology

Up to date anatomical and biochemistry studies related to Brain mechanisms of behavior are reviewed. This type of research shold be work up in clinical psychology related to prevention, diagnostic and therapy of behavior disorders. In this way, the clinical psychology should make clinical research at differents age groups of the population. He will be able to study cpidemiological risk factors together with neuropsychologycal tests and Brain imagind studies

Key words: psychophisiology, pshisiological psychology, biological basis of behavior

1. El doctor Edmundo Beteta es profesor principal de Neurología en la Facultad de Medicina y Jefe de Laboratorio de Psicofisiología en la Facultad de Psicología de la UNMSM. Realizó estudios de Post-grado en la Universidad de Miami, Florida; en Columbia University de Nueva York y en la Universidad Johns Hopkins, Baltimore. Fue presidente de la Sociedad Peruana de Psiquiatría, Neurología y Neurocirugía. Ha publicado 3 libros y 40 artículos en su especialidad, en revistas nacionales y extranjeras. Dirección: Faustino Sánchez Carrión 411, Lima-Perú, Fax: 431-5041. CE: d280003@unmsm.edu.pe. 

Los últimos estudios de anatomía, fisiología y bioquímica del cerebro han revelado interesantes correlaciones clínicas en el área de la conducta. El despistaje de factores biológicos de riesgo, en un vasto grupo de alteraciones de la conducta humana, así como, las investigaciones bioquímicas y el diagnóstico por imágenes (Resonancia Magnética Cerebral, Tomografía por emisión de positrones con estudios de flujo sanguíneo y metabolismo cerebral), han permitido progresar en nuevas perspectivas de la psicología clínica.

La experiencia de los psicológicos y neurólogos, en amplias casuísticas, han demostrado la interrelación entre los hallazgos de los tests neuropsicológicos y las anormalidades anatómicas y bioquímicas, descritas en los estudios por imágenes, así como por los exámenes neurofisiológicos (Electroencefalografía y potenciales evocados).

Este informe, presenta los últimos estudios anatómicos, neurofisiológicos y bioquímicos que permiten ampliar el campo de prevención, diagnóstico y tratamiento de los problemas de conducta, con el mayor conocimiento de los factores biológicos en el área de la psicología clínica.

\section{Estudios anatómicos}

Los estudios morfológicos y neuropatológicos, han demostrado la importancia del cerebelo en funciones cognitivas y también en la expresión de desórdenes afectivos (Schmanhman, 1991) dando lugar a un nuevo desorden descrito como el síndrome cognitivo-afectivo cerebeloso (Schmanhman, 1998). Igualmente, se han descrito variantes del tamaño del cerebelo y lóbulos cerebrales, controlados por estudios de Resonancia Magnética Cerebral (MRI), en relación con el desorden de hiperquinesia 
y déficit de atención (Berquin, 1998; Filipek, 1997), denominado síndrome de ADHD (Atention Deficit Hyperactivity Disorder). En fin, lo niños con autismo que presentan defectos de sociabilidad, lenguaje y comunicación asociados a retardo mental en muchos casos, también presentan anomalías en el desarrollo morfológico del cerebelo con reemplazo de neuronas de Purknje por gliosis, además, alteraciones de la estructura de la Oliva Bulbar (paleocerebelo) y otras anormalidades del tronco encefálico (Bayley, 1998; Raplin, 1998).

Se han comparado dos síndromes cromosómicos, caracterizados por alteraciones cognitivas y lingüísticas: el síndrome de Williams (deleción del cromosoma 7) y el síndrome de Down (trisomía del cromosoma 21). Se encontró que el cerebelo estaba dramáticamente reducido en el síndrome de Down y de tamaño normal en el síndrome de Williams (Jernigan, 1993). Sin embargo, en este último, se han podido demostrar severas alteraciones bioquímicas, consistentes en una disminución del marcador neuronal del aminoácido neutrasmisor $\mathrm{N}$ Acetil aspartato, de gran importancia en la performance de una variedad de tests cognitivos (Rae, 1998). Mediante los circuitos fronto subcorticales y los inputs del cerebelo al lóbulo frontal, se han descrito, un grupo de pacientes con agresividad y violencia en relación a lesiones del cerebelo, especialmente ubicadas en el vermis, de tal manera, que actualmente en neurofisiología, se propone que el neocerebelo, así como el neocortex, intervenga en coordinación de las funciones cognitivas, mientras que el paleocerebelo, así como el sistema límbico, se relacione con la expresión emocional (Cummings, 1993).

Los estudios anatómicos del cuerpo estriado y sus correlaciones neuropatológicas han demostrado, que además de liderar el movimiento automático, por sus componentes bioquímicos tiene interrelación con el área afectiva-volitiva. De este modo, diversas lesiones del núcleo candado y globus pallidus en un grupo de pacientes, determina el desorden obsesivo-compulsivo y estados anormales afines, tales como despersonalización, hipocondrías, anorexia nerviosa, compulsiones sexuales, tricotilomania, cleptomanía, tortícolis, síndrome de Tourette (enfermedad de los tics), aurismo, corea de Sydenham y diversos desórdenes impulsivos que pueden 96 
llegar a la violencia compulsiva (Cia, 1997; Cummings, 1993).

Mediante el análisis volumétrico de la Resonancia Magnética Cerebral y una batería de Tests Neuropsicológicos realizada en 15 pacientes de sexo masculino, portadores de síndrome de atención e hiperquinesia (Síndrome de $\mathrm{ADHD}$ ), se comprobaron anomalías estructurales en las regiones prefrontal-frontal derecha y sus conexiones bilaterales con el núcleo caudado, respondiendo a la hipótesis de una disfunción de la vía de la Dopamina fronto-estriada y la deshinbición del hemisferio derecho, resultado en síntomas de hiperactividad e impulsividad (Filipek, 1997).

Los adelantos en el estudio de la organización de la corteza cerebral, y la substancia blanca subcortical, en relación con diversos procesos patológicos, han demostrado que la organización columnar de la corteza (antiguamente se consideraba organización laminar), permite un sistema de distribución funcional versátil, por el cual, una lesión focal cortical puede degradar una función, pero de ningún modo alterarla totalmente. En la organización columnar, existen áreas corticales sensoriales-motrices que intervienen en cognición, percepción y personalidad (áreas hetereotípicas), y otras áreas que trabajan en toda la asociación de funciones (áreas homotípicas) (Mountcastle, 1997).

Desde el punto de vista funcional, las antiguas áreas numeradas de Brodman, han sido reemplazadas, por un grupo de áreas unimodales que responden básicamente a una sola información o una sola respuesta (áreas motoras-sensoriales), mientras que otras áreas responden a diferentes tipos de estimulaciones, generalmente localizadas en la región prefrontal y se denominan multimodales (áreas que procesan las estimulaciones del sistema límbico). De este modo, la interpretación de lesiones corticales puede variar, si compromete un sistema funcional cognitivo-perceptivo u otro sistema funcional que relacione personalidad y expresión afectiva, (Mesulman, 1998).

La revisión de la anatomía funcional de la substancia blanca subcortical revela que ocupa la mitad del volumen de los hemisferios cerebrales en 
el cerebro adulto. Asimismo, que la substancia blanca de los lóbulos frontales establece relaciones de asociación con todas las regiones del cerebro, es decir, que la proporción de substancias blanca es definitivamente mayor en el hemisferio cerebral derecho, especialmente en el lóbulo frontal. Esta consideración anatómica determina que las lesiones difusas de la substancia blanca son las que originan disturbios de los sistemas de atención, habilidades visoespaciales, funciones del lóbulo frontal y defectos emocionales, mientras que las habilidades lingüísticas están conservadas. Los desórdenes patológicos que afectan la substancia blanca (Esclerosis múltiple, leucoencefalopatías, enfermedad de Biswanger, contusión cerebral, leucodistrofia metacromática complejo demencial del SIDA y otros) producen demencia con pérdida cognitiva, desórdenes del estado afectivo, apatía, abulia, inatención, depresión y psicosis (Filley, 1998).

La investigación de factores biológicos en el desarrollo de la violencia (Beteta, 1992), ha sido estudiada en 2,306 casos de pacientes y criminales portadores de tres tipos de violencia: episódica por síndrome del descontrol, psicopática secundaria a lesiones orgánico-cerebrales y compulsiva en desórdenes compulsivos (Elliot, 1992). En esta casuística, como factores de riesgo para el desarrollo de violencia, se han encontrado disfunciones cerebrales en niños con problemas de aprendizaje, síndrome de ADHD, autismo, síndrome de Tourette, disfunciones del cerebelo y desórdenes obsesivo-compulsivos, casos de correlación anatómica-clínica ya descritos en párrafos anteriores.

La hipótesis en relación a que lesiones ventromediales del lóbulo frontal, incrementan el riesgo de conducta agresiva y violenta, ha sido confirmada en el estudio de 1,221 sobrevivientes de la guerra de Vietnam, entre 19671970, portadores de heridas penetrantes en el cráneo. El estudio realizado 15 años después en el centro médico del Ejército (Walter Reed Army Medical Center, Washington, D.C.), mediante pruebas neuropsicológicas, electroencefalograma, potenciales evocados, y Tomografía Cerebral, demostró en 520 traumatizados, una buena correlación entre los valores de la escala de agresión/violencia y las lesiones ventromediales del lóbulo frontal, en comparación con un grupo de sujetos control (Grafman, 1996). 
Entre los factores biológicos de riesgo, en el desarrollo de violencia (Beteta, 1992), también se han reportado pacientes con historia de epilepsia, especialmente la epilepsia del lóbulo temporal. La etiología de este tipo de epilepsia se relaciona con una predisposición genética que determina malformaciones del hipocampo y subsecuentemente, convulsiones febriles que terminan en la denominada esclerosis mesial de lóbulo temporal, un desorden anatómico-patológico caracterizado por muerte neuronal y su reemplazo por células de la glía, constituyéndose en un foco epileptogénico. Los estudios clínicos, han sido corroborados-mediante exámenes de electroencefalografía y resonancia magnética cerebral-, demostrándose la correlación entre la anormalidad bioeléctrica cerebral y la atrofia del hipocampo del lóbulo temporal, (Fernández, 1998; Hernán, 1997; Paesschen, 1997).

Por último, en el diagnóstico diferencial de episodios de violencia y epilepsia del lóbulo temporal, deben mencionarse los estados disoactivos del sueño y la vigilia. Al respecto, los estudios del Centro de Investigaciones en sueño de Minnesota (EUA) han reportado graves casos de violencia durante el estado de sueño que han terminado en homicidios y suicidios. Por tales motivos esta área de investigación ha cobrado gran importancia en psicología forense y en medicina legal (Ahowald, 1992).

\section{Estudios bioquímicos}

Están referidos a los defectos genéticos. Se ha encontrado que en diferente material cromosómico, pero especialmente en el cromosoma 21 , existe una correlación entre el síndrome de Down y la enfermedad de Alzheimer. En ambas entidades, se presenta un defecto de $\beta$-amiloide, la apolipoproteina E4 y la proteína TAU. El niño con Down tiene un envejecimiento prematuro y después de los 35 años desarrolla alteraciones patológicas en el cerebro que son la expresión clínica de la enfermedad de Alzheimer. Esto sugiere que la proteína anormal, asociada a un defecto del neurotrasmisor acetil colina, refleja un proceso de envejecimiento y demencia progresiva. Similar proteína, la synelfina, se ha encontrado en una 
variedad de canarios. Éstos sólo trinan cuando la proteína aumenta en el cerebro y cuando envejecen se constata la disminución de la synelfina y la pérdida del trino (George, 1998).

En el autismo se ha podido encontrar un defecto de la serotonina (Raplin, 1998). Por otro lado, en casos de violencia se ha demostrado experimental y clínicamente la alteración del equilibrio entre serotonina y vasopresina (Cummings, 1993).

Actualmente por medio de la tomografía de emisión de positrones (PET), se ha demostrado, en un grupo de asesinos violentos que no presentaban deprivación psicosocial, un defecto en el metabolismo de la glucosa en la región prefrontal (Raine, 1998). El avance bioquímico más importante está referido al área inmunológica. Se ha podido demostrar, en niños que tuvieron antecedentes de infección bacteriana por estreptococo, que un porcentaje de ellos ha desarrollado síndromes de déficit de atención (Síndrome de ADHD), múltiples tics del tipo Tourette, movimientos involuntarios de tipo coreico y estereotipias (Kurlan, 1998; Singer, 1998). Mediante estudios bioquímicos utilizando los tests de ELISA y Western Blots (similares a los tests para diagnosticar SIDA), se pudieron definir anticuerpos (que fueron anticuerpos antibacterianos), que tenían predisposición para lesionar estructuras cerebrales, es decir anticuerpos anticerebro, dando lugar a diversos desórdenes cerebrales que pueden lesionar la estructura neuronal, la mielina, las proteínas receptoras de las sinapsis y los neurotrasmisores. Estos desórdenes producen alteraciones de las funciones cognitivo-afectivas, al lado de perturbaciones motoras severas como ocurre en la esclerosis múltiple, enfermedad que destruye la mielina del sistema nervioso central en pacientes jóvenes (Hoelfeld, 1997).

\section{Psicofisiología y psicología clínica}

La psicofisiología, también denominada Psicología Fisiológica, referida igualmente a las bases biológicas de la conducta, está dedicada al conocimiento de los mecanismos cerebrales que soportan la conducta humana, la experiencia y la conciencia. En este objetivo, subtiende a la 100 
Psicología que es la ciencia de la conducta y la experiencia y usufructa los estudios de la etología y la psicología experimental.

Se han revisado los aportes anatómicos y bioquímicos de los últimos años que magnifican el conocimiento de los mecanismos cerebrales. En este sentido, el psicólogo clínico y el neurólogo deben realizar investigaciones del mayor interés tanto en el área de la prevención como en el terreno diagnóstico y terapéutico.

En la actualidad, en el área de epidemiología deben tomarse en cuenta los factores de riesgo biológico en desórdenes de la conducta, y despistarse en grupos poblacionales, especialmente en niños y adolescentes. A continuación deben llevarse a cabo estudios neuropsicológicos complementados con exámenes radiológicos de imágenes cerebrales. De este modo, los profesionales de las neurociencias podrán ayudar de modo más certero y eficaz a la comunidad.

La revisión de psicofisiología presentada, no excluye los estudios de psicología social y educacional; por el contrario, se complementa con las investigaciones clínicas. De este modo, el estudio de los pacientes y el despistaje de los aparentemente normales, se realiza de modo interdisciplinario y por lo tanto, pragmático.

Las investigaciones de psicofisiología clínica, publicadas en múltiples revistas de psicología en EUA y Europa, de ningún modo deben anublar la creatividad de los especialistas que defienden la influencia del ambiente social en el desarrollo de los problemas de conducta. Tampoco deben descartarse, en el binomio organización cerebral-medio ambiente, las hipótesis clásicas de Pavlov y Luria en relación a la conceptualización de la personalidad y la conciencia. En fin, se debe considerar la dualidad aristotélica de cuerpo y alma, todo lo cual pertenece al campo de la heurística. 


\section{Referencias}

Bayley, A., Luther, P., Dean, A., Harding, B., Janota, I., Montgomery, M., Rutter, M. y Lantos, P. (1998). A clinic pathological study of autism. Brain, 121, 889-905.

Beteta, E. (1992). Neurobiología de la violencia. Boletín de la sociedad Peruana de Neurología, 3 (3), 116-120.

Berquin, P.C., Giedd, J.N., Jacobsen, L.K., Hamburgers, S.D. y Castellanos, F.X., (1998). Cerebellum in attention deficit Hiperactivity disorder. A morphometric MRI Study. Neurology 50, 1087-1093.

Cia, A.H. (1997). Trastorno obsesivo-compulsivo y sindromes relacionados. Ponencia presentada en IV Congreso Internacional de Psiquiatría. Simposio internacional sobre nuevos desarrollos en trastornos del espectro obsesivo compulsivo. Buenos Aires, Argentina.

Cummings, J.L. (1993). Frontal subcortical circuits and human behavior. Arch Neurol, 50, 873-880.

Elliot, F.A. (1992). Violence the neurologic contribution: An Overview. Arch Neurol, 49, 595-603.

Fernández G., Effenberger, O., Vinz, B., Steinlein, O., Elger, C.E., Dohring $y$ W. Heinze, H.J. (1998). Hippocampal malformation as a cause of familiar febrile convulsions and subsequent hippocampal sclerosis, Neurology, 50, 909-917.

Filipek, P.A., Semurd-Clikeman, M., Steingard, R.J., Renshaw, P.F., Kennedy, D.N. y Biderman, J. (1997). Volumetric MRI analysis comparing subjects having attention deficit hyperactivity disorder with normal controls, Neurology, 50, 1535-1540.

Filley, C.M. (1998). The behavioral neurology of cerebral white matter. Neurology, 50, 1535-1540.

George Julia M. y Clayton, D.F. (1998). Song birds, synelfin and neurodegerative disease. Neuroscience News, 1, 2; 12-17.

Grafman, J., Schwab, K., Warden, D., Pridgen, A., Brown, H.R. y Salazar, A.M. (1996). Frontal lobe injuries, violence and aggression: A report of the Vietnam head injury study. Neurology, 46, 1231-1238.

Herman, B.P., Seidernberg, M., Shoenfeld, J. y Davies, K. (1997). Neuropsychological charactristic of the syndrome of mesial temporal 102 
La psicofisiología y el desarrollo de la Psicología Clínica

lobe epilepsy. Arch Neurol, 54, 369-376.

Hoelfeld, R. (1997). Biotechmological agents for the inmuno therapy of multiple sclerosis principles, problems and perspectives. Brain, 120, 865-916.

Jernigan, T.L., Bellugi, U., Soweli, E. Doherty, S. y Hesselink, J.R. (1993).

Cerebral morphologic distinctions between Willians and Down syndromes. Arch Neurol, 50, 186-191.

Kurlan, R. (1998). Tourette's syndroe and "PANDAS" will the relation bearout. Neurology, 50, 1530-1543.

Mahowald, M.W., Shenck, C.H., Rosen, G.M. y Hurwtiz, T.D. (1992).

The role of a sleep violence. Arch Neurol, 49, 604-607.

Mesulman, M.M. (1998). From sensation to cognition. Brain, 121, 10131052.

Mountcastle, V.B. (1997). The columar organization of the neurocortex. Brain, 120, 701-722.

Paesschen, W.V., Revesz, T. Duncan, J.S. King, M.D. y Conelly, A. (1997).

Quantitive neuropathology an quantitive magnetic resomance imaging o the hipocampus in temporal lobe epilepsy. Ann. Neurol, 42, 756-766.

Raine, A., Stoddard, J., Bihrle, S. y Buchsbaum, M. (1998). Prefrontal glucose deficit in marderers lacking psychosocial deprivation. Neuropsychiatri, Neuropsycology and behavioral Neurology, 11, 1-7.

Raplin, I. y Katzman, R. (1998). Neurobiology of atism. Ann. Neurol, 43, 7-14.

Rae, C., Karmiloff-Smith, A., Lee, M.A., Dixon, R.M., Grant, J., Blamire, A.M., Thompson, C.H., Styles y P. Radda, G.K. (1998). Brain biochemeistry in william syndrome evidence for role of the cebellum in cognitivion. Neurology, 51, 33-40.

Schmanhman, J.D., Sherman, J.C. (1998). The cerebellar cognitive affective syndrome. Brain, 121, 561-579.

Schmanhman, J.D. (1991). An emerging concept The cerebellar contribution to higer finction. Arch Neurol, 48, 1178-1187.

Singer, H.S., Giuliano, J.D., Hansen, B.H., Hallety, J.J., Laurino, J.P., Benson, M. y Kiessling, L.S. (1998). An atibodies against human putament in children with Tourette syndrome. Neurology, 50, 1618-1624. 